\title{
La statistique médicale de la FMH en tant qu'instrument de la planification des besoins?
}

Voir aussi l'éditorial du Dr Daniel Herren, membre du Comité central de la $\mathrm{FMH}$, concernant la statistique médicale de la FMH.
Correspondance: Esther Kraft

$\mathrm{FMH}$

Département DDQ Elfenstrasse 18

CH-3000 Berne 15

Tél. 0313591111

Fax 0313591112

ddq@fmh.ch

\author{
L'opinion d'une scientifique, d'un politicien et d'un médecin de famille \\ A: Pourquoi le système de santé suisse a-t-il besoin de chiffres fiables et significatifs? \\ B: Pourquoi une base de données solide est-elle importante pour le corps médical? \\ C: Quelle importance donnez-vous à la statistique médicale de la FMH en tant qu'instrument \\ de planification des besoins?
}

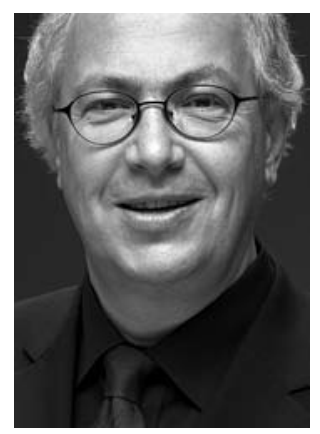

Thomas Burgener

Conseiller d'Etat du canton du Valais, chef du Département de la santé, des affaires sociales et de l'énergie

A: Une nouvelle réglementation de l'admission à pratiquer des fournisseurs de prestations doit être élaborée d'ici au 31 décembre 2009, c'està-dire avant que le gel des admissions ne prenne fin. Par ailleurs, les assureurs maladie soutiennent l'introduction de la liberté de contracter mais, pour ma part, je rejette la levée de l'obligation de contracter car il faut cesser de renforcer le pouvoir des caisses. Les cantons et la FMH ont elaboré une solution de rechange consistant à maintenir le principe de l'obligation de contracter, tout en donnant la possibilité aux cantons d'intervenir au moyen de mesures concrètes en cas de pénurie ou de pléthore de médecins. Reste à convaincre les Chambres fédérales qui semblent pour l'heure préférer un système basé sur la liberté de contracter.

Indépendamment de cette réglementation, des chiffres statistiques fiables et concluants sont nécessaires pour garantir à la population un approvisionnement correct en soins.

B: L'expérience faite avec la limitation de l'admission des fournisseurs de prestations a montré qu'il est très important de disposer de données statistiques solides pour pouvoir réguler l'offre de manière satisfaisante, aussi bien pour les patients que pour les fournisseurs de prestations. Le taux d'occupation des médecins, les disciplines effectivement pratiquées et la qualité des prestations n'ont jamais été recensés jusqu'à présent. En outre, le développement des cabinets de groupe exerce une pression sur la réglementation actuelle.

A l'avenir, il faudra des données précises en suffisance pour pouvoir améliorer le système de dosage de l'offre et éviter un sur- ou un sous- approvisionnement au niveau de chaque région en particulier.

C: La statistique médicale de la FMH est un instrument très significatif et d'une grande importance pour les autorités cantonales de la santé. Elle permet de recenser en continu le nombre de médecins praticiens par canton et par discipline. Elle sert en outre de base pour différentes études destinées à mesurer l'offre médicale d'après le taux d'occupation des médecins et la spécialisation qu'ils pratiquent effectivement.

En l'an 2000, nous avons institué dans notre canton un observatoire valaisan de la santé. Ce système d'information a pour tâche de saisir la totalité des données de santé actuelles (domaine ambulatoire et hospitalier). A cet égard, la statistique médicale de la FMH est une source de données absolument indispensable.

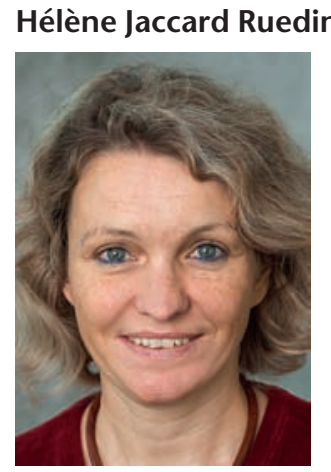

Cheffe de projet, I'Obsan

A: L'accès aux soins est un droit fondamental de chaque citoyen. Il implique une certaine planification des infrastructures et des ressources. Pour en assurer le financement, il nécessite en outre que des mesures de régulation soient mises en place. Pour cela, des données de qualité sont nécessaires à différents niveaux:

- En renseignant sur les besoins de la population et sur les ressources à mettre en œuvre pour répondre à ces besoins, elles facilitent la planification, la régulation et l'organisation du système de santé de manière à en garantir l'accessibilité.

- En fournissant des indications sur la composition des coûts des soins, elles contribuent à comprendre la dynamique du système . 
Finalement, en décrivant l'évolution du système, elles permettent d'évaluer l'impact des mesures de régulation prises.

B: Pour les médecins, l'intérêt de données de qualité varie selon le domaine qu'elles décrivent:

- Les données décrivant les besoins de la population et leur évolution contribuent à la recherche épidémiologique. Elles permettent d'adapter l'offre en soins et d'évaluer l'impact de services médicaux fournis, comme par exemple des mesures préventives.

- Les données décrivant les services utilisés/ fournis renseignent sur les ressources à engager pour répondre à un certain besoin. Elles deviennent un outil de base dans la recherche médicale consacrée à la qualité de la prise en charge.

- Dans un autre registre, les données décrivant les ressources disponibles ont été utilisées pour réguler les effectifs. Dans ce cas, des données de qualité permettent d'éviter les erreurs d'interprétation.

C: La nouvelle statistique FMH décrit justement le corps médical de manière plus détaillée. Les effectifs de médecins sont différenciés selon le lieu d'activité, le taux d'activité ou selon certaines activités de base. Elle renseignera de manière plus réaliste sur les différents types de services médicaux disponibles, à un échelon régional. En suivant l'évolution de l'offre, il sera possible d'évaluer l'impact des tendances qui se dessinent au sein du corps médical et de mesures politiques incitatives ou restrictives.

Elle facilitera aussi les projections quant aux besoins futurs en médecins nécessaires dans les différents secteurs.

Cependant, il importe de préciser qu'elle permettra de réaliser des projections et non des planifications. Cette dernière démarche, réalisée par exemple par les «Health Systems» anglosaxons, nécessite des données beaucoup plus détaillées.

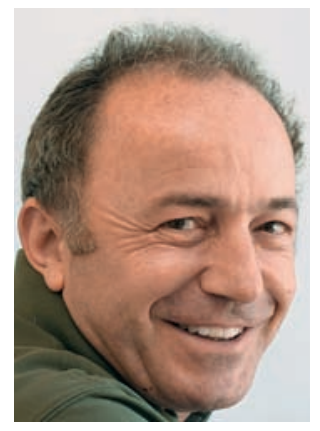

Franz Marty

Médecin de famille à Coire, médecin spécialiste en médecine générale $\mathrm{FMH}$

A: Il est nécessaire de disposer de chiffres concluants pour
- élaborer une documentation fiable sur les champs d'intérêt actuels

- analyser les questions qui se posent

- établir des bilans

- disposer de bases valables pour chaque planification.

Le défi de taille, surtout dans le domaine de la santé, c'est de détecter les données essentielles dans un océan de chiffres. Il est donc important d'avoir des indicateurs indépendants, solides, d'un coût avantageux et faciles à saisir.

B: Des données solides sont importantes dans tous les domaines, qu'ils soient professionnels ou administratifs: dans notre activité quotidienne, nous nous appuyons sur des données de laboratoire précises, regroupons nos factures (données de prestations) pour en faire une base de données utiles; enfin, la recherche et l'enseignement reposent sur des données éloquentes.

Précisément dans le domaine de l'indemnisation des prestations fournies, ces dernières années ont montré que la survie économique du médecin de famille indépendant ne peut guère être garantie sans des données solidement documentées (centres fiduciaires).

C: La nouvelle statistique médicale de la FMH fournit pour la première fois des chiffres documentés sur les médecins pratiquant la médecine de famille. La saisie du taux d'occupation permet de tirer des conclusions sur les effectifs réels de ces médecins. La statistique prend désormais aussi en compte le type de cabinet (individuel ou de groupe).

Comme la charge administrative est limitée à un minimum, on peut espérer que les membres l'accepteront largement et participeront en grand nombre à la saisie des données. La nouvelle statistique médicale de la FMH sera certainement un outil important de l'évaluation des besoins et, pour les médecins de famille, une amélioration notoire. La connaissance des forces de travail à disposition n'est toutefois qu'un côté de la médaille, les besoins en médecine de famille de la société (nouvelles offres thérapeutiques, démographie) en sont l'autre et constituent une valeur de référence qui reste à déterminer. 